\title{
DOI: https://doi.org/10.24297/jaa.v11i.8861
}

\section{Design of multilayer microfluidic paper chip and its visual detection of pesticide residues}

\author{
Li zhi Li ${ }^{1 *}$, Han Ping Mao ${ }^{1}$
}

${ }^{1}$ Key Laboratory of Modern Agricultural Equipment and Technology, Ministry of Education, Jiangsu University, Zhenjiang, China

maohp@ujs.edu.cn

\begin{abstract}
With the increase of China's grain production, the use of pesticides is gradually increasing. Traditional pesticide detection takes a long time and requires expensive experimental instruments, which is not conducive to the rapid and accurate detection of pesticide residues in the field. To solve this problem, this paper proposes a visual detection method of pesticide residues based on multi-layer microfluidic paper chips. The internal channel structure of paper chip is designed from the perspective of efficient mixing. Through the simulation of the mixed effect of three kinds of staggered channel structures, which are arc type, triangle type, and ladder type, the "ladder-type h-0.3, s-2.6" is selected as the best-staggered structure, and the mixing strength is 0.91534 . The best simulation structure was tested by a colored reagent, and the image processing of 15 test results was carried out with MATLAB. The average mixing strength was 0.84 , and the and the standard deviation was 0.022 . The visual detection experiment of acetamiprid and profenofos in cabbage samples was carried out by using the device, The detection range of acetamiprid was $4 \sim 72 \mu \mathrm{g} / \mathrm{kg}$, and the detection range of profenofos was $3 \sim 54 \mu \mathrm{g} / \mathrm{kg}$. The recovery of acetamiprid was $75 \% \sim 85 \%$, and the recovery of profenofos was $80 \% \sim 90 \%$. The detection range and recovery rate indicate that the device has high repeatability and accuracy in the actual sample detection
\end{abstract}

Keywords: facility horticulture, crop growth information, intelligent detection equipment, multi-sensor detection system, information fusion

\section{Introduction}

The traditional methods of pesticide residues detection include chromatography1 [1] and spectroscopy [2]. The traditional methods have high accuracy, but the detection process is time-consuming, requiring hundreds of thousands of instruments or even millions of professional personnel .The traditional detection methods can not detect pesticide residues in real time quickly and easily. However, the widely used rapid detection card can detect pesticides in real time, but the detection limit is $0.3 \sim 3.5 \mathrm{mg} / \mathrm{kg}$. The detection limit of pesticide residues in fruits and vegetables was higher than $\mu \mathrm{g} / \mathrm{kg} \sim \mathrm{mg} / \mathrm{kg}$, so the detection quantity of the quick detection card is higher than that of the human body. The cost of paper-based microfluidic chip is lower than that of glass, PDMS, quartz and other materials because it is based on paper. Moreover, without external driving, the sample, reagent and waste liquid in the fluidic chip are micro, with high integration, low consumption and easy to carry. The detection of pesticide based on paper microfluidic chip is greatly convenient for the detection personnel and is conducive to the real-time detection on site.

CLémence et al.[5] made a two-dimensional paper chip by means of wax spray printing, which was used to detect organophosphate in water. Martinez [3] and others for the first time demonstrated the stacking design of 3D $\mu$ pads. The paper chips were stacked together by double-sided adhesive tape, and the hydrophobic channel was made by photolithography. Holes were drilled on the double-sided adhesive to ensure the 
connection between the upper and lower layers, so as to obtain the ability to move fluid in a three-dimensional manner. Liu et al.[4] for the first time prepared upads by three-dimensional origami method, and verified its applicability by color detection of glucose and protein. However, the channels in these three kinds of paper chips are all simple straight channels, and there is no channel shape designed to make mixing more efficient. In order to make different reactants fully mix and react, this paper designs different channel structures based on three-dimensional paper chip, and studies the mixing effect of different structures.

In this paper, we use the intrinsic fluorescence effect between ratio fluorescent quantum dots and gold nanoparticles. At the same time, coupled with the appropriate ligand technology, the pesticide residue detection is transformed into the detection of color change visible to the naked eye. A three-dimensional multi-layer array paper chip was designed, and the structure of the mixing zone was optimized by simulation and experimental research, so as to improve the mixing efficiency and the visual detection accuracy of pesticides.

\section{Design of multilayer microfluidic paper chip}

\subsection{Overall design of multilayer paper chip}

In order to meet the requirements of rapid and convenient detection of a variety of pesticides, a foldable multilayer paper-based microfluidic chip was designed, and the three-dimensional paper chip was made by wax spray printing method [7]. The required pattern was printed on the surface of filter paper by wax spray printer, and then heated to melt the wax. The melted wax penetrates down into the paper to form hydrophobic channels.

Fig. 1 (a) is a 15 piece of nine palace paper channel plan designed by using AutoCAD. Nine groups of samples can be injected at the same time. Each layer consists of nine $6 \times 6 \mathrm{~cm}$ squares with the same structure, and these squares are composed of $3 \times 3$ small squares with side length of $2 \mathrm{~cm}$. In addition to the white channel in the middle of the paper, all of them are hydrophobic areas formed by wax penetration. The white area is the channel with radius $r$ of $1.5 \mathrm{~mm}$ as the fluid flow mixing area.

Fig. 1 (b) is the schematic diagram of single sample detection in Fig. 1 (a).The three-dimensional paper chip is composed of five parts. The first layer is the first injection layer. The pesticide flows directly to the second injection layer through six single circular paper channels. The remaining two are the injection areas of gold nanoparticles and sodium chloride solution. The next area is the mixing area of the two reagents and there are six layers in the mixing zone. Hydrophobic regions of various shapes are printed on the circular channels of these six layers to form a structural channel to promote the mixing of gold nanoparticles (AuNPs) and sodium chloride $(\mathrm{NaCl})$. After mixing, $\mathrm{NaCl}$ makes AuNPs completely agglomerate, and the mixture of the two liquids also enters the second injection area to mix with the pesticide.The eighth layer is the second injection area, in which there is a control channel for sodium chloride and gold nano solution to enter alone. The channel is a single circular channel, which is used to make the mixture flow to the control area of the final detection layer and react with the modified substrate to form a detection control.

Six layers below the eighth layer are all mixed layers, which are used to mix the mixed solution of sodium chloride and gold nanoparticles with pesticides. Pesticides of different concentrations can weaken the agglomeration of gold nanoparticles. The last layer, namely the 15 th layer, is the detection layer. The detection area and the control area are designed, and the ratio fluorescent quantum dots are modified on the detection layer. The gold nanoparticles with different agglomeration degree will react differently with the quantum dots Color fluorescence. 


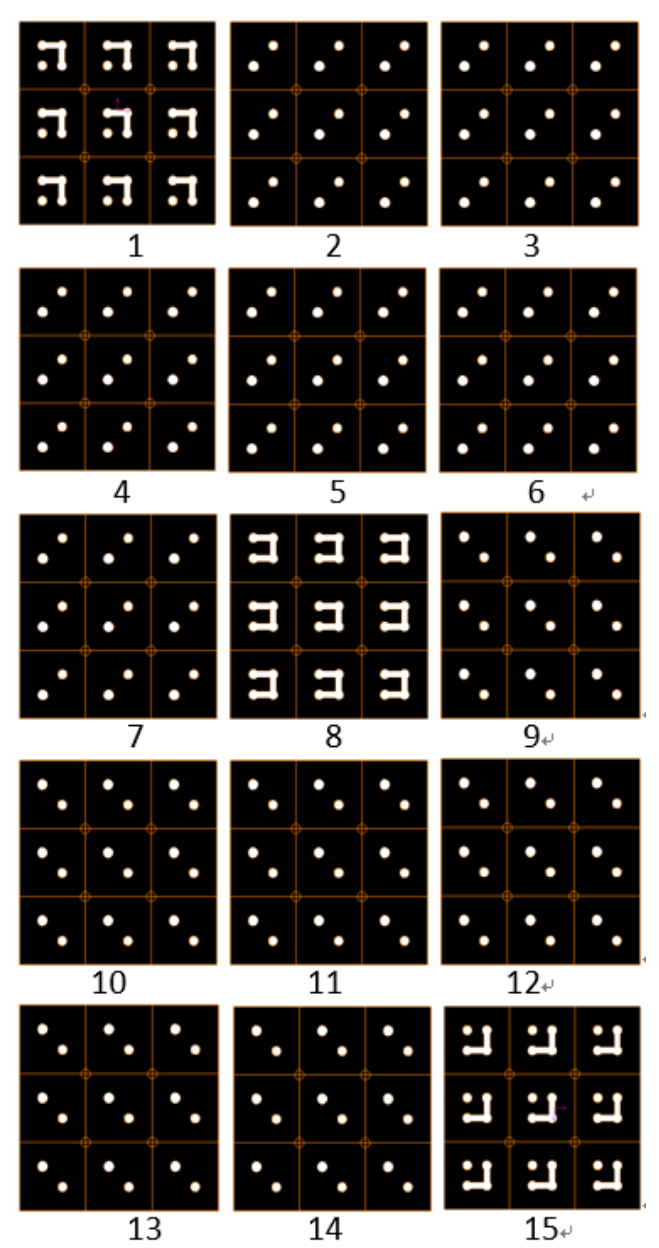

(a)

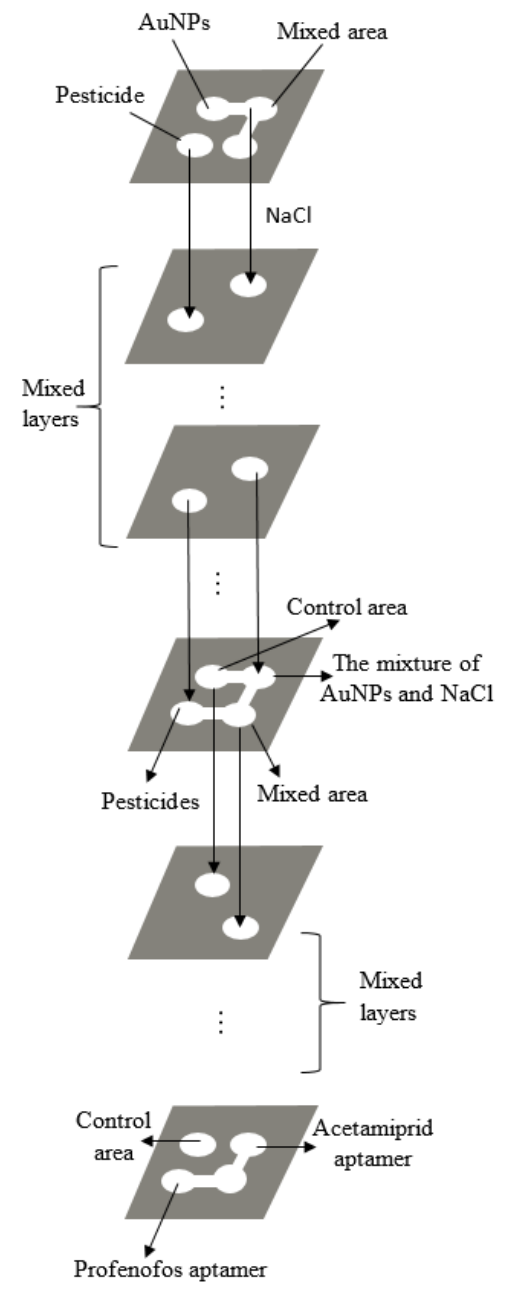

(b)

Fig 1. Design of paper chip and folding sequence

The fluid transportation in paper is a passive process, which is realized by the capillary action of paper. Through folding, 3D paper chip enables multiple flow layers to penetrate liquid layer by layer. In addition to the flow in each layer of paper, it can also flow vertically between two layers. This method enables the fluid to be transported to a large number of reaction sites in a compact area.

\subsection{Structure design of mixed channel}

The chip channel shown in Fig.1 (b) has two mixing regions. Both samples are mixed through six layers of mixing layer. When the two sample solutions are mixed in the mixing layer, there is no third solution involved in mixing, so the two mixing regions choose the same mixing structure. When the structure of the mixing zone is optimized and simulated by COMSOL software, a total of 8 layers of paper channel from sample injection to mixing end is selected for simulation.

The two basic mechanisms of fluid mixing are diffusion and convection. In order to make two or more fluids mix well without any external driving equipment, it is necessary to design the internal channel shape reasonably. Different channel shapes make the transported fluid stretch, compress, separate and converge to achieve the 
mixing effect. When the structure of the mixing zone is optimized by COMSOL software, a total of 8 paper channels from sample injection to mixing end are selected for simulation, and the middle six layers are mixed layer. Figure 2 shows the simulation diagram of two simulated liquids mixing on the 8-layer paper chip.

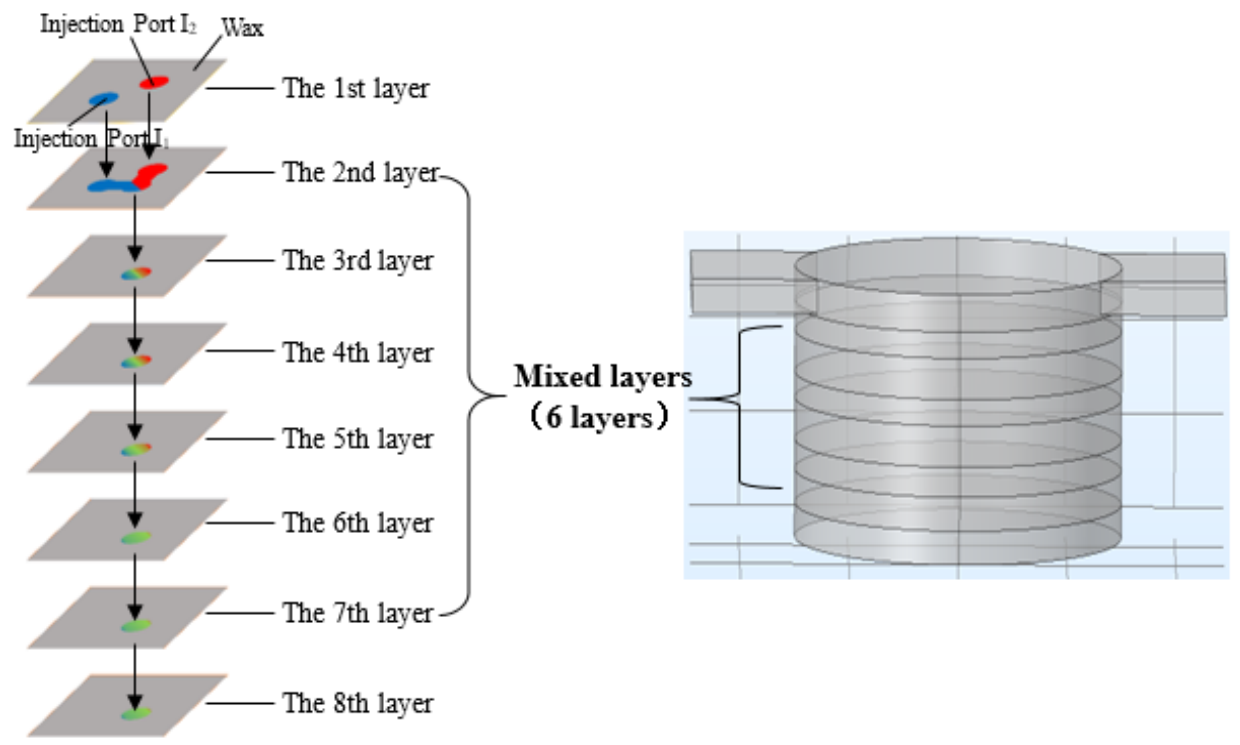

Fig 2. Schematic diagram of single sample detection of two pesticides on the paper channel

According to the mixing principle of fluid tension and compression, three kinds of three-dimensional inner rib structures are designed in this paper: circular arc (Fig.3), triangular (Fig.4) and ladder (Fig.5). Fig.6 shows the cross section of circular arc, triangular and ladder channels respectively.

As shown in Table 1, determine the side length "s" of the ladder type as $1.4 \mathrm{~mm}$, change the factors $\mathrm{H}_{1}, \mathrm{H}_{2}$ and $\mathrm{H}_{3}$, and select four parameter values of each factor for simulation to determine the relationship between parameters and mixing performance, select $\mathrm{H}_{1}, \mathrm{H}_{2}, \mathrm{H}_{3}$ that can make the best mixing effect, and then select four $\mathrm{s}$ parameters with selected $\mathrm{H}_{3}$ to further determine the parameter size of ladder structure. The vertical channel radius $R$ is $1.5 \mathrm{~mm}$ and the height $h$ is $1.44 \mathrm{~mm}$ (there are eight layers of paper in the mixing zone, and the thickness of each layer is about $0.18 \mathrm{~mm}$ ).
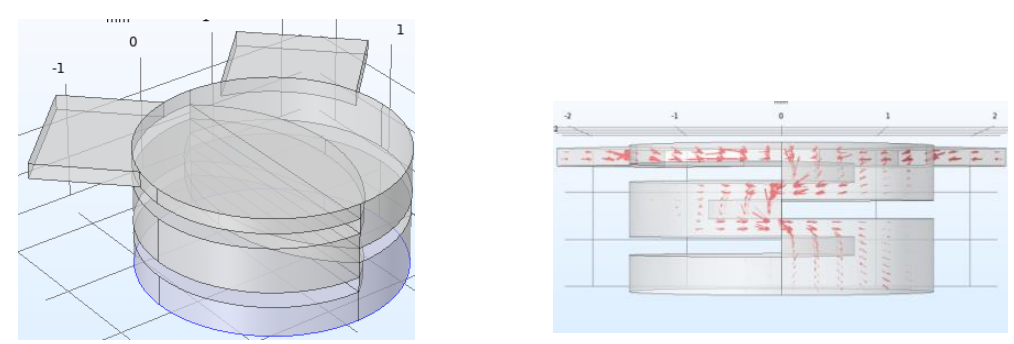

Fig 3 . Circular structure diagram 

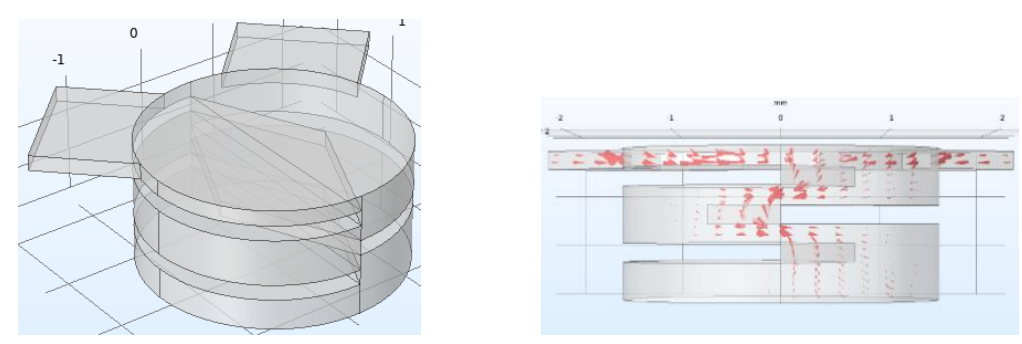

Fig 4 .Triangle structure diagram
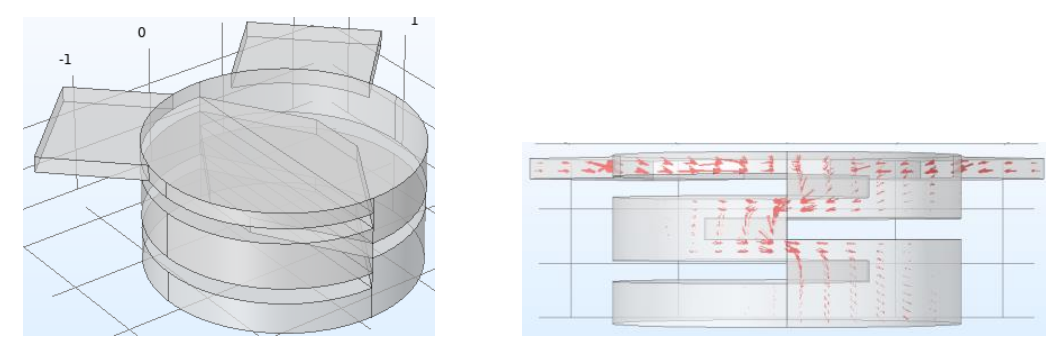

Fig 5 . Ladder structure diagram
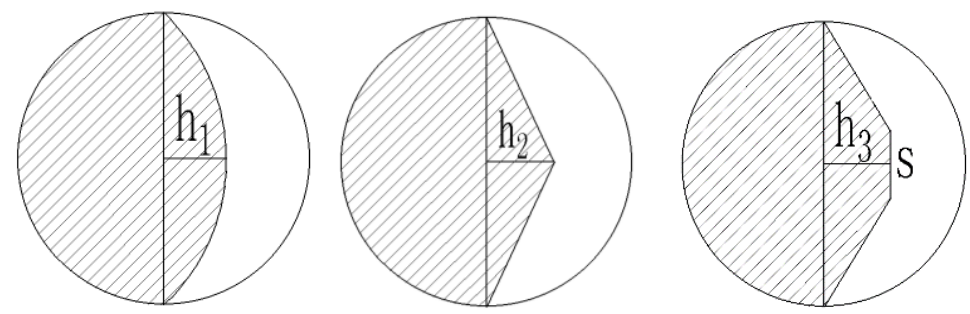

Fig 6 . Sectional view of 3 flow channels

Table1 Main parameters and selected values of pipe wall obstacle structure

\begin{tabular}{llllll}
\hline Structures & Parameter & \multicolumn{4}{l}{ Parameter value $(\mathbf{m m})$} \\
\hline Circular arc & $\mathrm{h}_{1}$ & 0 & 0.3 & 0.6 & 0.9 \\
Triangular & $\mathrm{h}_{2}$ & 0 & 0.3 & 0.6 & 0.9 \\
Ladder & $\mathrm{h}_{3}$ & 0 & 0.3 & 0.6 & 0.9 \\
Ladder & $\mathrm{S}$ & 0.8 & 1.4 & 2 & 2.6 \\
\hline
\end{tabular}

2 Results and analysis

\subsection{Flow mixing simulation of three structures}

\subsubsection{Mixed effect evaluation index}

The mixed strength value ie can be used to evaluate the mixing effect, and the value ie of the mixing strength 
is defined as le[8]:

$$
I_{e}=\operatorname{aveop}\left(1-a b s\left(c_{i}-\bar{c}\right) / \bar{c}\right)
$$

aveop() is the average operator in $\mathrm{COMSOL}_{\text {; }}$ abs() is the absolute value;

$c_{i}$ is the concentration at a point on the cross section $(\mathrm{mol} / \mathrm{L}) ;$

$\bar{c}$ is the average concentration of the cross section ( $\mathrm{mol} / \mathrm{L})$.

$\mathrm{I}_{\mathrm{e}}$ is a dimensionless value in the range of $0 \sim 1$. When it is 1 , it means that the mixture is sufficient, and when it is 0 , it means that there is no mixing.

\subsubsection{Simulation results of three hybrid structures}

Fig. 7, Fig. 8 and Fig. 9 show the comparison diagram of mixed strength $l_{e}$ at the exit of each floor of arc type, triangle type and ladder type structure $(s=1.4 \mathrm{~mm})$ with parameters $\mathrm{h}$ of $0 \mathrm{~mm}, 0.3 \mathrm{~mm}, 0.6 \mathrm{~mm}$ and $0.9 \mathrm{~mm}$. In addition, the blank structure without any channel shape was set as the control group. The results showed that even though the structures were different, the mixing effect was better with the decrease of $h$. However, when $\mathrm{h}$ was reduced to $0 \mathrm{~mm}$, the mixing effect became worse when $\mathrm{h}$ was $0.3 \mathrm{~mm}$. Therefore, when $\mathrm{h}=0.3 \mathrm{~mm}$, the mixing effect of the last layer of the three structures was the best, ie was $0.86679\left(\mathrm{H}_{1}=0.3 \mathrm{~mm}\right), 0.85846\left(\mathrm{~h}_{2}=\right.$ $0.3 \mathrm{~mm})$ and $0.86679\left(\mathrm{H}_{1}=0.3 \mathrm{~mm}\right)$, respectively $88374\left(\mathrm{H}_{3}\right.$ is $\left.0.3 \mathrm{~mm}\right)$, so the ladder with $\mathrm{H}_{3}$ of $0.3 \mathrm{~mm}$ is selected to continue the optimization simulation.

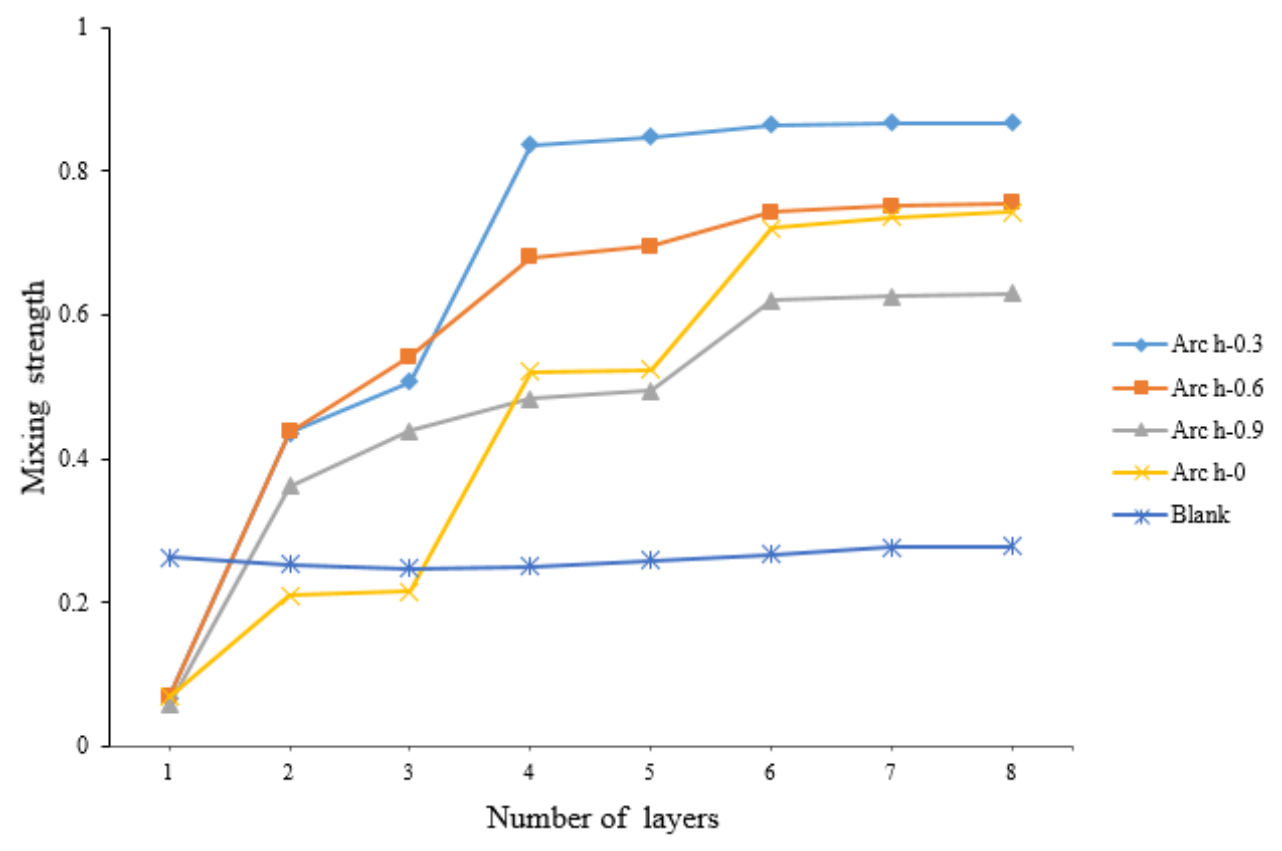

Fig 7. Mixed strength value of circular structure 


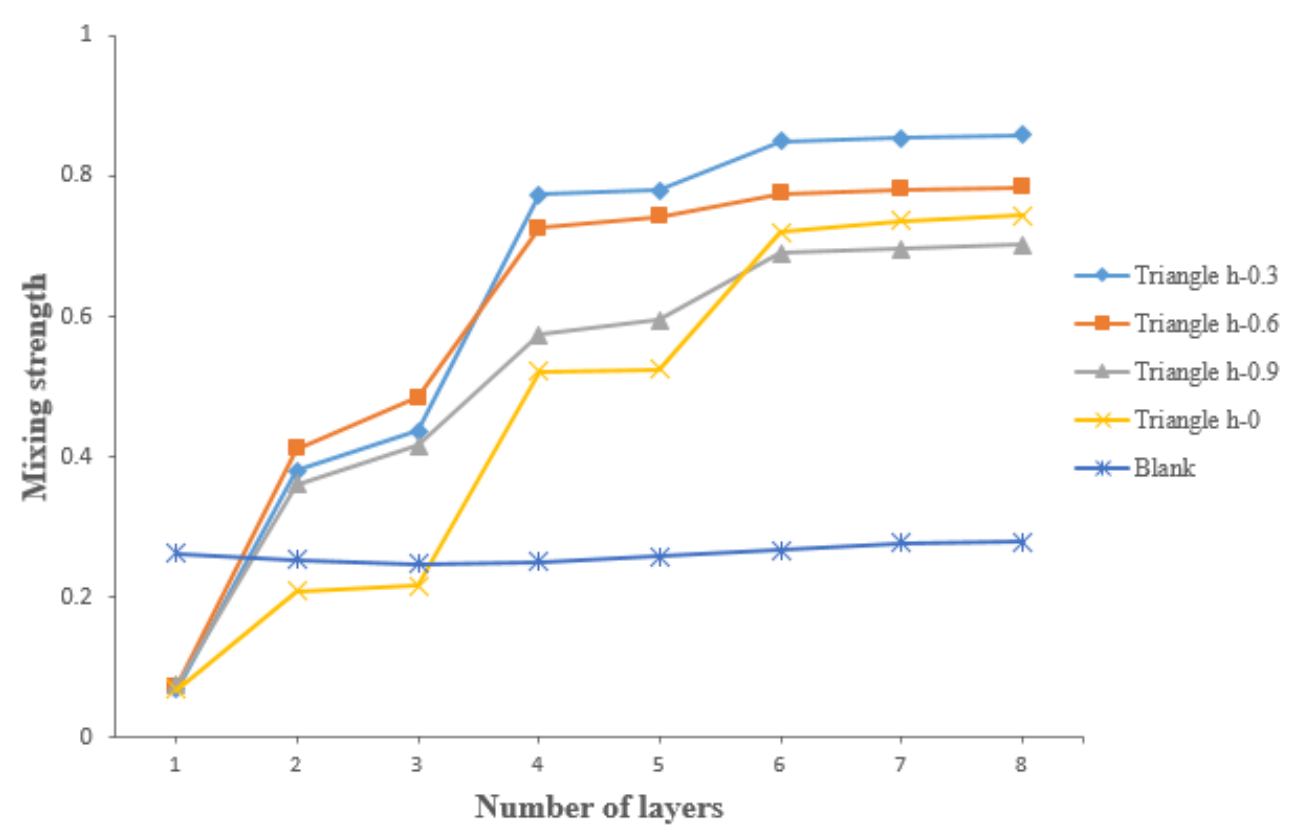

Fig 8 . Mixed strength value of triangle structure

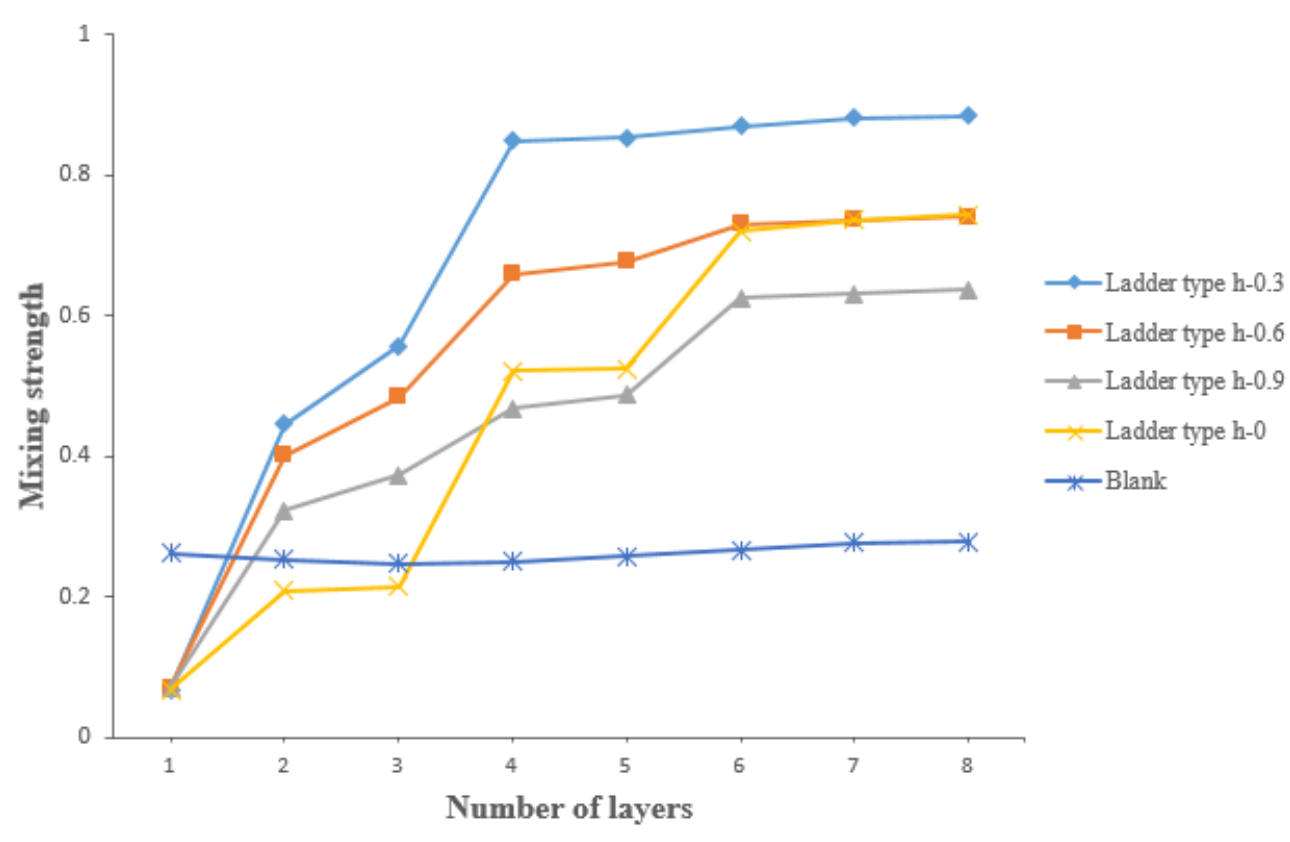

Fig 9 .Mixed strength value of ladder structure

Fig. 10 shows the contrast diagram of the mixing strength $\mathrm{l}_{\mathrm{e}}$ at the exit of each layer after changing the length $\mathrm{s}$ of the upper and bottom to extend or compress towards the two sides of the channel while keeping $\mathrm{H}_{3}$ at 0.3 . The results show that the higher the mixing strength is, the greater the mixing strength is. When $\mathrm{s}$ is $2.6 \mathrm{~mm}$, the maximum mixing strength $\mathrm{l}_{\mathrm{e}}$ is 0.91534 . Therefore, the ladder structure with $\mathrm{H}_{3}$ of $0.3 \mathrm{~mm}$ and $\mathrm{s}$ of $2.6 \mathrm{~mm}$ is selected as the optimal three-dimensional inner rib structure to design the flow channel, and this structure is recorded as "ladder h-0.3,s-2.6". 


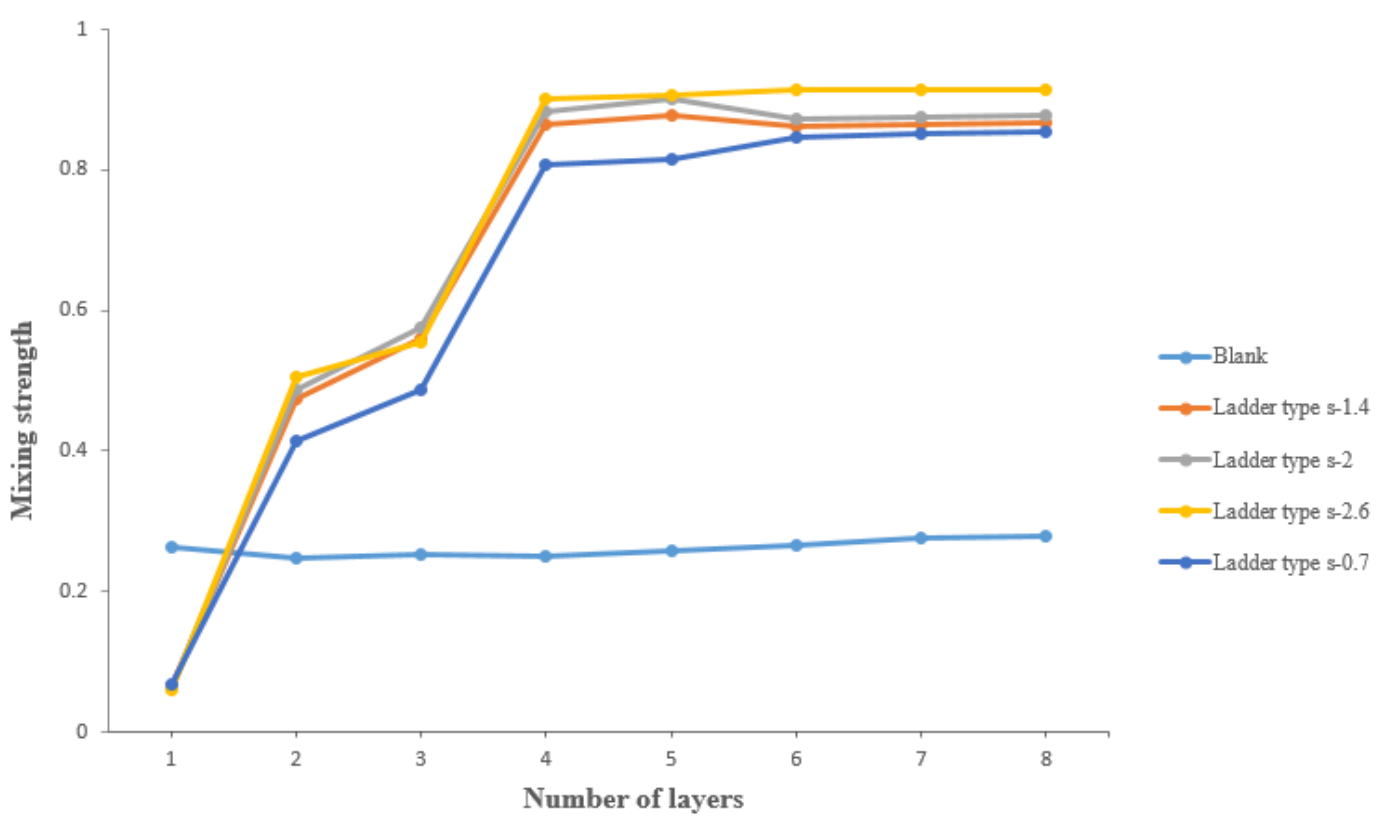

Fig 10 .The mixed strength value of the best parameters of trapezoidal structure with different $s$

Compared with the above results, the analysis is as follows:

In our selected values, when $\mathrm{h}$ is $0.3 \mathrm{~mm}$, the simulation effect is the best. When $\mathrm{h}$ is $0.9 \mathrm{~mm}$, the interlaced structure is obvious, which will cause great interference to the velocity of the fluid. However, if the interference exceeds a certain limit, it will hinder the diffusion movement of the fluid, and make the fluid molecules unable to exchange and diffuse better, resulting in poor mixing effect. On the other hand, from the perspective of different shapes, the ladder shape is the best, followed by arc type and triangle type. It is speculated that the shape of ladder shape makes the fluid more intense folding and expansion on the basis of ensuring diffusion, so as to achieve better mixing effect. When $\mathrm{s}$ is $2.6 \mathrm{~mm}$ in the ladder structure, the flow velocity and direction of the fluid on both sides of the channel are well changed, resulting in a greater degree of tensile deformation, so that the mixing effect is better and the mixing strength is greater.

\subsection{Multi layer paper chip mixing experiment}

Two solutions of methyl orange and methyl blue were selected as the mixed solution and dropped into the 3D paper chip as shown in Fig.11a. At the same time, the control experiment of blank structure (Fig. 12b) was set. As shown in FIG. 11, FIG. 11C shows the flow mixing of each layer of "ladder h-0.3, s-2.6", and Fig. 11d shows the mixing of each layer in the blank structure control group. The color uniformity of Fig. $11 \mathrm{C}$ is better than that of Fig. $11 \mathrm{~d}$. 

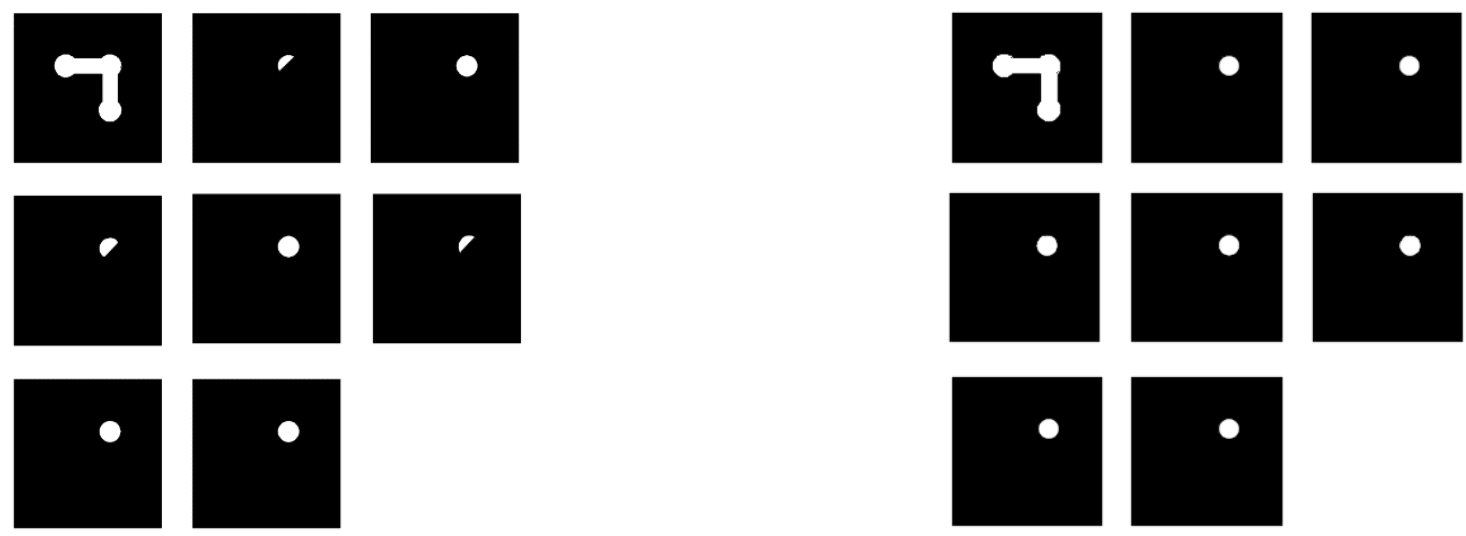

b. blank structure diagram

a. "ladder h-0.3,s-2.6" structure diagram
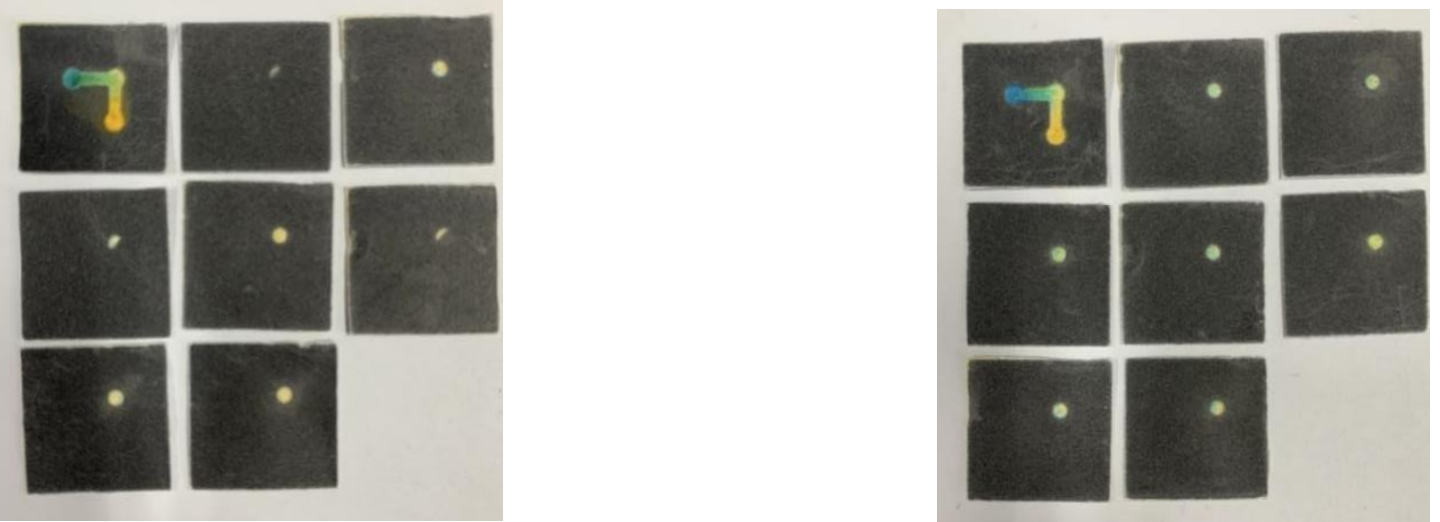

\section{c. Mixed effect photo of "ladder h-0.3,s-2.6" structure}

d. Mixed effect photo of blank structure

\section{Fig 11 Effect diagram of mixed contrast test}

The mixing effect of multi-layer paper-based microfluidic chips was evaluated by image processing. Define the value of mixing strength ie as

$$
\mathrm{I}_{e}=1-\frac{1}{\bar{c}} \sqrt{\frac{\sum_{i=1}^{N}\left(c_{i}-\bar{c}\right)^{2}}{N}}
$$

Where $\mathrm{N}$ is the number of pixels in the measurement area;

$c_{i}$ is the gray value of each pixel;

\section{$\bar{c}$ is the average gray value}

$\mathrm{I}_{\mathrm{e}}$ is a dimensionless value in the range of $0 \sim 1$. When it is 1 , it means that the mixture is sufficient. When it is 0, it means that there is no mixing. Matlab is used to extract the gray value of each pixel in the yellow area in Fig.12a, and the average value is calculated. Finally, the gray value $c_{i}$ of the pixel points in the mixed region in Figure 12 is extracted. The mixed intensity value is 0.84 calculated by formula (2). The mixing experiment was repeated for 15 times. After each mixing, the cross-section of the channel outlet of the micromixer was extracted by camera in turn. The mixing intensity diagram was calculated by the above method, as shown in FIG. 13 . The average value of mixing strength calculated by 15 tests was 0.85 , and the standard deviation was 0.022 . 


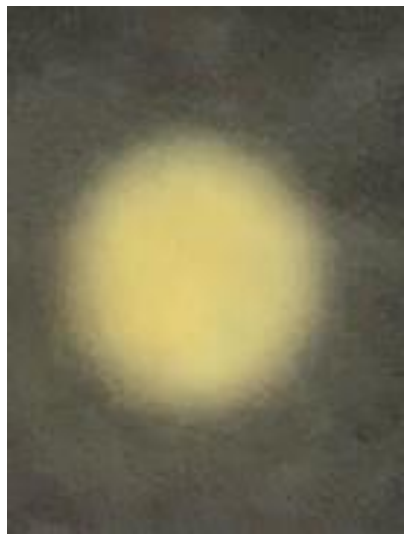

(a)

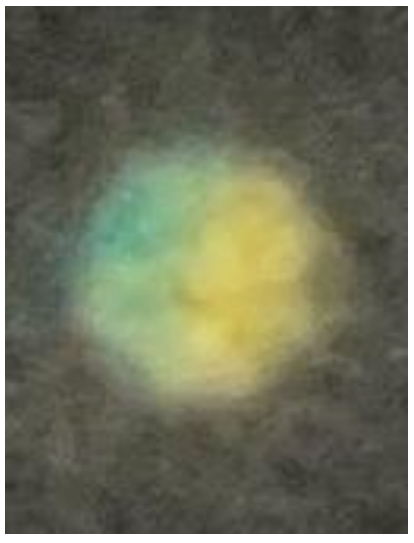

(b)

Fig 12 Experimental photos of two kinds of structured paper chips

Comparing the experimental results with the simulation results, it is found that the trend of the calculation results is consistent with the simulation results. The experimental results show that the designed structure effectively solves the problem of uniform mixing of the two fluids.

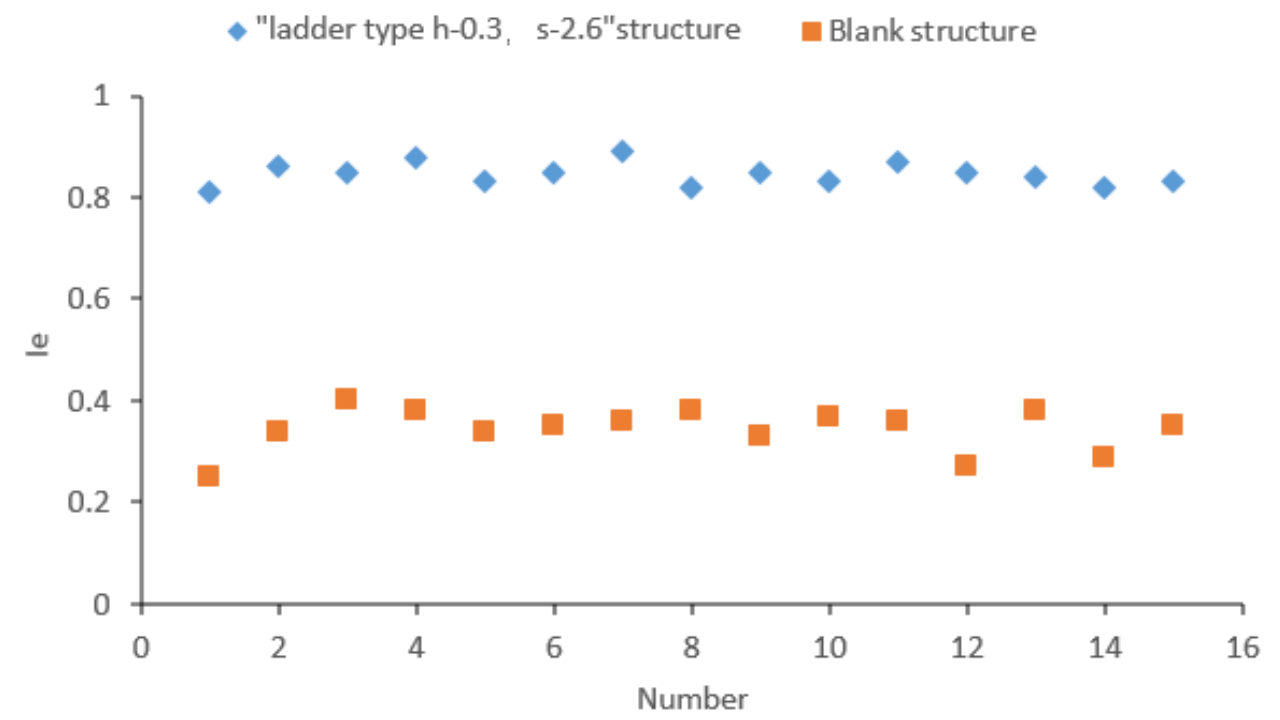

Fig 13 Comparison of mixing strength between two kinds of structured paper chips

\subsection{Pesticide detection experiment of array 3D paper chip}

The principle of pesticide detection: the green part of the ratio fluorescent quantum dots and gold nanoparticles produce fluorescence internal efficiency, and the green fluorescence is quenched; when AuNPs are in sodium chloride solution, the aggregation between AuNPs occurs, which weakens the fluorescence internal filtering effect between the ratio fluorescence probe and AuNPs, and the fluorescence color of green quantum dots in the ratio fluorescence probe is restored.

When the aptamer of acetamiprid is added, it will be adsorbed on the surface of AuNPs due to the coordination between aptamer and AuNPs, thus enhancing the salt out resistance of AuNPs and dispersing the agglomerated AuNPs again. As a result, the fluorescence internal filtering effect between the ratio fluorescent probe and AuNPs should be enhanced, and the fluorescence intensity of the green quantum dots of the ratio fluorescent probe will decrease. When acetamiprid is added, the aptamer fell off the AuNPs due to the specific binding between 
the aptamer and acetamiprid, and AuNPs were induced to agglomerate again by sodium chloride, which led to the decrease of fluorescence internal filtering effect and the green quantum dots fluorescence color of ratio fluorescent probe was restored again. Therefore, the concentration of acetamiprid determines the intensity of fluorescence internal filtering effect, and the fluorescence intensity of ratio fluorescence quantum dots is related to the concentration of acetamiprid, thus realizing the visual detection of pesticide residues.

The visual detection experiment of acetamiprid and Profenofos in cabbage samples was carried out. Fig.14 shows the fluorescence response of Acetamiprid in the detection area under different concentrations $(0 \mu \mathrm{g} / \mathrm{kg}$, $4 \mu \mathrm{g} / \mathrm{kg}, 8 \mu \mathrm{g} / \mathrm{kg}, 14 \mu \mathrm{g} / \mathrm{kg}, 24 \mu \mathrm{g} / \mathrm{kg}, 32 \mu \mathrm{g} / \mathrm{kg}, 44 \mu \mathrm{g} / \mathrm{kg}, 56 \mu \mathrm{g} / \mathrm{kg}, 72 \mu \mathrm{g} / \mathrm{kg}$ ) of Acetamiprid in the detection area. With the increase of acetamiprid concentration, the fluorescence color of the detection area changes from pink to orange to yellow to yellow to green. This series of visible color changes show that by taking photos and then extracting RGB with MATLAB, the relationship between $b$ value and acetamiprid pesticide concentration is established (FIG. 15). The linear equation is $B_{1}=0.69 c_{1}+115, B_{1}$ is the corresponding $b$ value of RGB in the photo, $C_{1}$ is acetamiprid. The detection range was $4 \sim 72 \mu \mathrm{g} / \mathrm{kg}, R_{2}$ is 0.9226 .

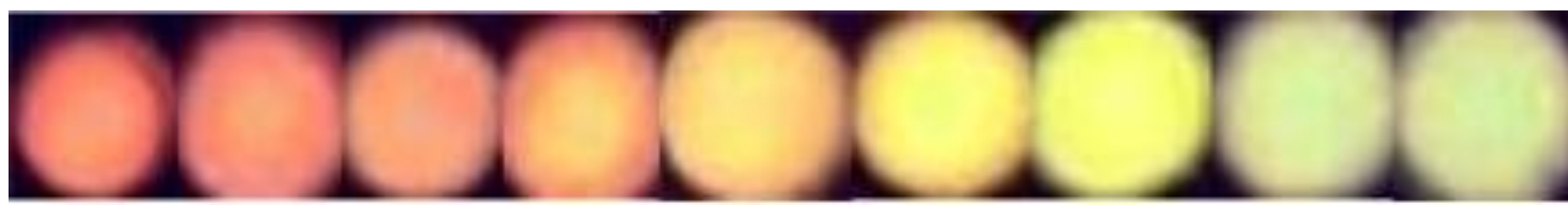

Fig 14 Flourescence photos of acetamiprid at different concentrations on paper

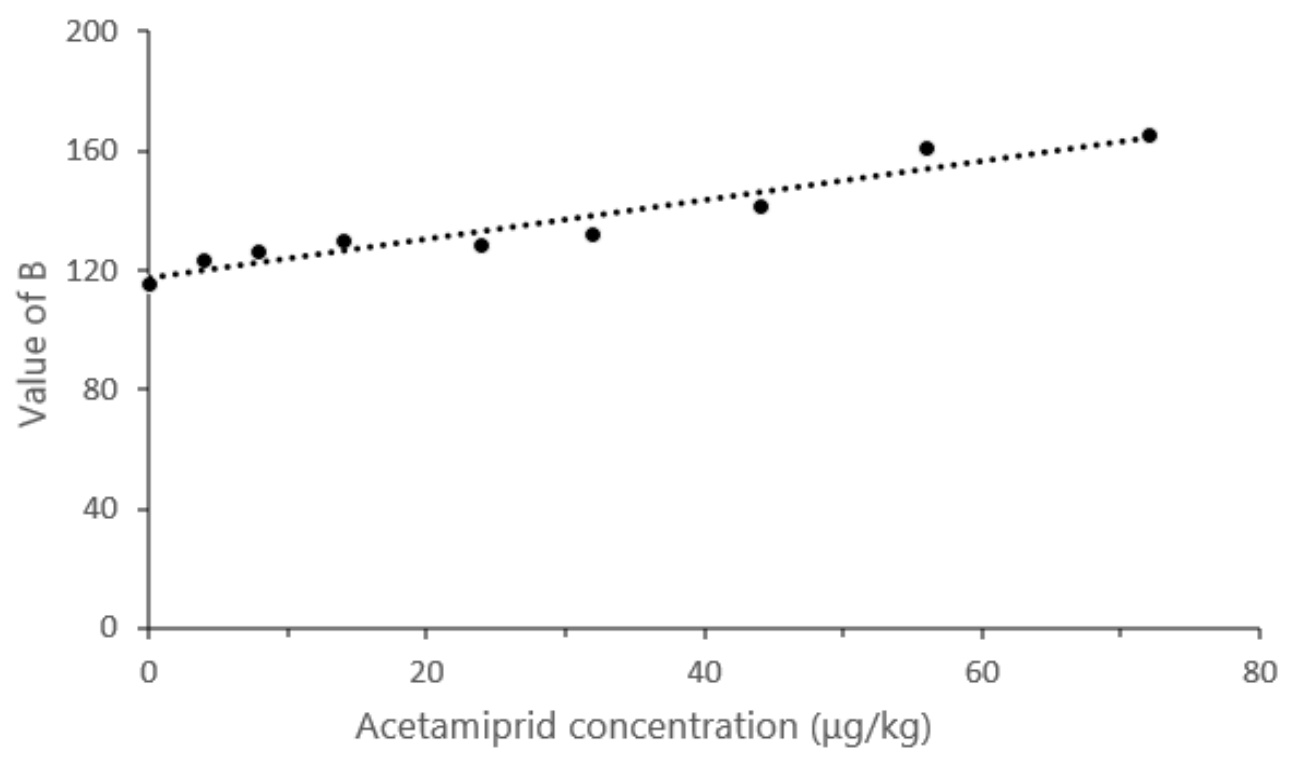

Fig 15 The relationship between the concentration of acetamiprid and the concentration of acetamiprid on paper

Different concentrations of profenofos solution were dripped into the solution respectively. Fig. 16 shows the fluorescence color change photos of the ratio fluorescence sensing system of profenofos with different concentrations ( $0 \mu \mathrm{g} / \mathrm{kg}, 3 \mu \mathrm{g} / \mathrm{kg}, 6 \mu \mathrm{g} / \mathrm{kg}, 12 \mu \mathrm{g} / \mathrm{kg}, 20 \mu \mathrm{g} / \mathrm{kg}, 28 \mu \mathrm{g} / \mathrm{kg}, 36 \mu \mathrm{g} / \mathrm{kg}, 46 \mu \mathrm{g} / \mathrm{kg}, 54 \mu \mathrm{g} / \mathrm{kg}$ ) under UV lamp. Figure 17 shows the relationship between $b$ value and pesticide concentration, the detection range is 3 $\sim 54 \mu \mathrm{g} / \mathrm{kg}$, the linear equation is $B_{2}=c_{2}+117, R_{2}$ is $0.9319, B_{2}$ is $R G B$ value, $c_{2}$ is profenofos concentration. 


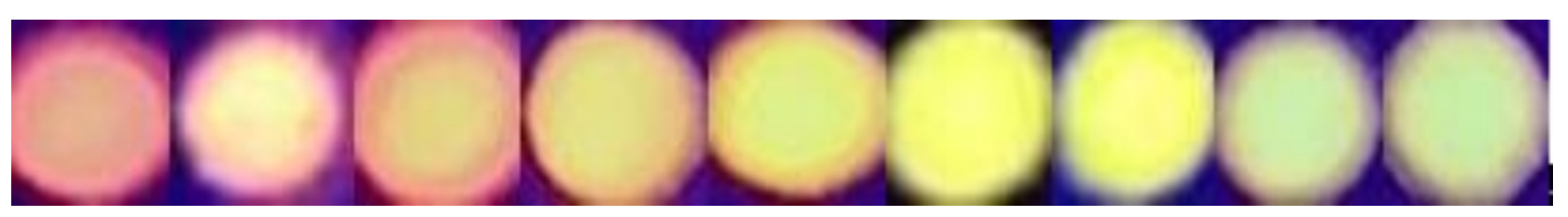

Fig 16 Detection of Profenofos in different concentrations on paper

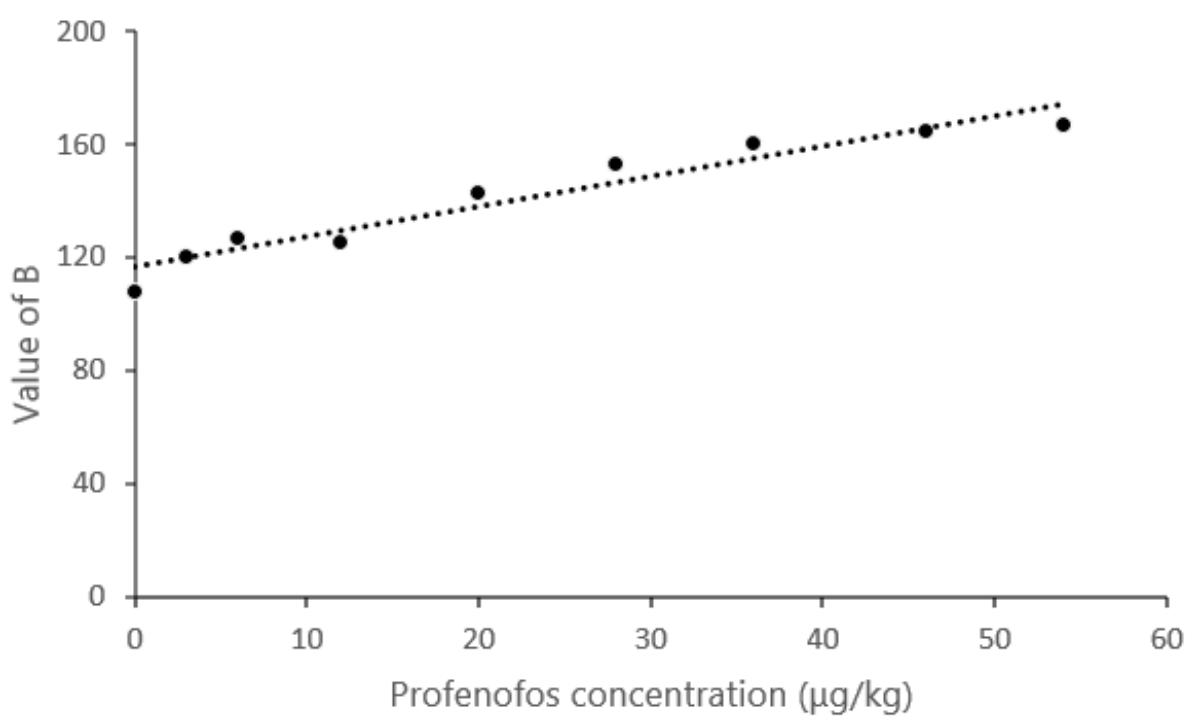

Fig 17 The relationship between the concentration of profenofos on paper and $b$ value

The detection range of acetamiprid was $4 \sim 72 \mu \mathrm{g} / \mathrm{kg}$ and that of profenofos was $3 \sim 54 \mu \mathrm{g} / \mathrm{kg}$. Both of them were lower than the national standard of $0.01 \sim 0.1 \mathrm{mg} / \mathrm{kg}$ of profenofos and Acetamiprid in vegetables. In order to verify the reliability of the device for pesticide detection, cabbage samples injected with acetamiprid and profenofos were detected by the device. As shown in Table 2 and table 3, the recoveries of acetamiprid and profenofos were $75 \% \sim 85 \%$ and $80 \% \sim 90 \%$, respectively, indicating that the device had high repeatability and accuracy in actual sample detection.

Table 2 Recovery of acetamiprid in chinese cabbage

\begin{tabular}{llll}
\hline Sample & Addition amount & Recovery & $\begin{array}{c}\text { (Response rate } \pm \text { RSD)(\%) } \\
(\mathbf{n}=\mathbf{3})\end{array}$ \\
\hline & 6.8 & 5.15 & $75.8 \pm 1.21$ \\
Chinese cabbage & 14.97 & $80.5 \pm 2.32$ \\
& & & \\
& 35.6 & 30.1176 & $84.6 \pm 1.45$ \\
\hline Table 3 Recovery of profenofos in chinese cabbage
\end{tabular}




\begin{tabular}{|c|c|c|c|}
\hline & 5.4 & 4.36 & $80.8 \pm 1.51$ \\
\hline \multicolumn{4}{|l|}{ Chinese cabbage } \\
\hline & 15.6 & 13.18 & $84.5 \pm 3.22$ \\
\hline & 20.4 & 17.87 & $87.6 \pm 2.25$ \\
\hline
\end{tabular}

\section{Summary}

1) A stacked multi-layer paper-based microfluidic chip was designed for the simultaneous detection of acetamiprid and Profenofos in nine samples. This structure enables the fluid to be transported to a large number of reaction sites in a compact area.

2) The results show that the "ladder h-0.3, s-2.6" structure has the best mixing effect, and the maximum mixing strength le is 0.91534 . A test is designed to verify the simulation results, and the trend of the two results is consistent.

3) The detection range of acetamiprid and profenofos was $4 \sim 72 \mu \mathrm{g} / \mathrm{kg}$, the linear equation was $B_{1}=0.69 c_{1}+115, R_{2}$ was 0.9226 , the detection range of Profenofos in cabbage was $3 \sim 54 \mu \mathrm{g} / \mathrm{kg}$, the linear equation was $B_{2}=C_{2}+117$, The $R_{2}$ was 0.9319 , which was lower than the national standard of $0.01 \sim 0.1$ $\mathrm{mg} / \mathrm{kg}$. The recovery rate of acetamiprid and profenofos was $75 \% \sim 85 \%$ and $80 \% \sim 90 \%$, respectively, which indicated that the device had high repeatability and accuracy in the actual sample detection.

\section{References}

1. A S F , A J M , A M C , et al. Simultaneous determination of 15 pesticide residues in Chinese cabbage and cucumber by liquid chromatography-tandem mass spectrometry utilizing online turbulent flow chromatography[J]. Food ence and Human Wellness, 2020.González F J E, Vidal J L M, Cano M L C, et al. Levels of metamidophos in air and vegetables after greenhouse applications by gas chromatography[J]. Journal of Chromatography A, 1998, 829(1-2): 251-258.

2. Liu H, Crooks R M. Three-Dimensional Paper Microfluidic Devices Assembled Using the Principles of Origami[J]. Journal of the American Chemical Society, 2011, 133(44):17564-17566.

3. Santhiago $M$, Henry C S, Kubota L T. Low cost, simple three dimensional electrochemical paper-based analytical device for determination of p-nitrophenol[J]. Electrochimica Acta, 2014, 130: 771-777.

4. Sicard C, Glen C, Aubie B, et al. Tools for water quality monitoring and mapping using paper-based sensors and cell phones[J]. Water research, 2015, 70: 360-369.

5. Sicard C, Glen C, Aubie B, et al. Tools for water quality monitoring and mapping using paper-based sensors and cell phones[J]. Water research, 2015, 70: 360-369.

6. Lu Y, Shi W, Jiang L, et al. Rapid prototyping of paper-based microfluidics with wax for low-cost, portable bioassay[J]. Electrophoresis, 2009, 30(9): 1497-1500.

7. Lee $Y K$, Tabeling $P$, Shih $C$, et al. Characterization of a MEMS-fabricated mixing device $[C]$. International mechanical engineering Congress et exposition. Orlando, Florida. 2000: 505-511.

8. Qin, J. W., Thomas, F., Burks, W. Gordon Bonn.(2009). Detection of citrus canker using hyperspectral reflectance imaging with spectral information divergence. Journal of Food Engineering. 93(2), 183-191 DOI:10.1016/j.jfoodeng.2009.01.014 